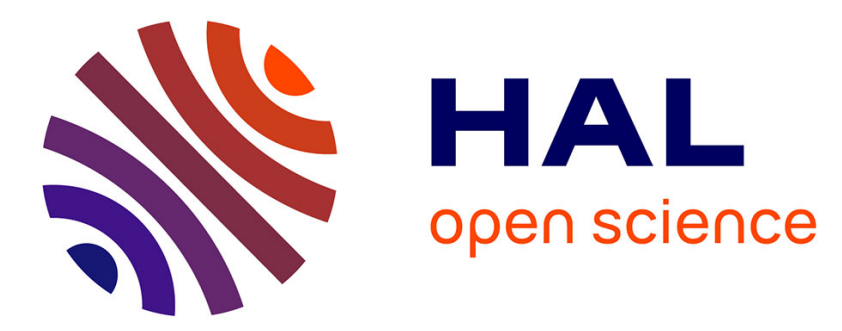

\title{
CURVATURE-BASED INTERFACE RESOLUTION QUALITY (IRQ) INDICATOR TO ASSESS SIMULATION ACCURACY
}

Romain Canu, Benjamin Duret, Julien Reveillon, François-Xavier Demoulin

\section{- To cite this version:}

Romain Canu, Benjamin Duret, Julien Reveillon, François-Xavier Demoulin. CURVATURE-BASED INTERFACE RESOLUTION QUALITY (IRQ) INDICATOR TO ASSESS SIMULATION ACCURACY. Atomization and Sprays, 2020, 30 (1), pp.31-53. 10.1615/AtomizSpr.2020033923 . hal02877689

\section{HAL Id: hal-02877689 \\ https://hal.science/hal-02877689}

Submitted on 22 Jun 2020

HAL is a multi-disciplinary open access archive for the deposit and dissemination of scientific research documents, whether they are published or not. The documents may come from teaching and research institutions in France or abroad, or from public or private research centers.
L'archive ouverte pluridisciplinaire HAL, est destinée au dépôt et à la diffusion de documents scientifiques de niveau recherche, publiés ou non, émanant des établissements d'enseignement et de recherche français ou étrangers, des laboratoires publics ou privés. 


\title{
CURVATURE-BASED INTERFACE
} RESOLUTION QUALITY (IRQ) INDICATOR TO ASSESS SIMULATION ACCURACY

\author{
R. Canu, ${ }^{1}$ B. Duret, ${ }^{2, *}$ J. Reveillon, ${ }^{3}$ E F.X. Demoulin ${ }^{4}$ \\ CORIA-UMR 6614 - Normandie Université, CNRS-Université et INSA de \\ Rouen, Campus Universitaire du Madrillet, 76800 Saint Etienne du Rouvray, \\ France.
}

*Address all correspondence to: B. Duret, E-mail: duret@coria.fr

Original Manuscript Submitted: 02/13/2020; Final Draft Received: mm/dd/yyyy

From the last decades direct numerical simulations of liquid-gas flows with interface capturing/tracking method have made great progress. But to address new configurations that contains highly deformed interfaces, question arises about the validity of the obtained results. Usually, the only solution is to perform a mesh convergence analysis on a well-defined criteria/quantity, which is really costly. For complex simulations such as liquid jet atomization, there is no evidence that a complete resolution of the interface deformation has been achieved, even if these simulations are still very helpful to understand atomization mechanisms. Thus it stresses the need to find an indicator dedicated to the evaluation of the Interface Resolution Quality (IRQ).

In this work, a definition for the IRQ indicator is discussed and computed in two configurations: a Two-phase HIT and the Spray A configuration (liquid jet injection) from the Engine Combustion Network. The first configuration is used as a reference to ensure the pertinence of the resolution indicator in an academic scenario, where the convergence of the main features of the flows is assured. Regarding the second configuration, the IRQ statistics such as PDF and average values of the IRQ are shown to assess its usefulness in a complex configuration for different Weber and Reynolds numbers.

KEY WORDS: DNS, interface, two-phas2 flow 
This document is a preprint version of the article. The published version is available in the Atomization and Sprays journal :

DOI: 10.1615/AtomizSpr.2020033923

5 1. INTRODUCTION

Liquid-gas flows occurs in many fields such as liquid fuel injection, plunging waves, cavitation, sloshing, pharmaceutical spray, painting, phase change, heat transfer, pipeline of two-phase flows, etc. For instance, liquid-gas flows have a direct impact on gas emissions (atomization in combustion engines), industrial process efficiency (heat exchanger) and coastal engineering (breaking waves). Many studies are dedicated to this kind of flows either experimentally or numerically. Despite important progresses that have been made recently, experimental results are particularly challenging to obtain in a turbulent two-phase environment. Regarding numerical developments, a lot of efforts has been made in the past decade to improve the description of the interface in different numerical formalisms, such as Large Eddy Simulation (LES) and Direct Numerical Simulation (DNS). Using DNS gives an access to almost all the scales of turbulent liquid-gas flows and allows a fine description of the interface behavior. The term "almost" used in the last sentence is important: up to now there is no equivalent theory for the smallest length scale as it can be defined in single phase flow for the Kolmogorov lenght scale or the Batchelor length scale.

Since the first studies on atomization DNS (Ménard et al. (2007), Desjardins et al. (2008), Herrmann (2008)), the question of the required resolution to assess that a simulation is a "true DNS" is still unanswered. As mentioned in Gorokhovski and Herrmann (2008), few DNS studies illustrate their grid dependency with a grid convergence analysis. A discussion on the smallest scale observable in a two-phase flows DNS is also present, arguing that reaching a true two-phase DNS resolution is more expensive than its single-phase counterpart, due to breakup events leading to liquid structures becoming smaller and smaller. Later, Shinjo and Umemura (2010) performed a computation with 6 billion points and despite this important effort in terms of mesh resolution, it is not clear that the smallest scale has been captured with the finest mesh. Nevertheless, there has been numerous successful studies combining direct numerical simulations (DNS) of the flow dynamic and a resolved interface. There is no doubt that these studies are

\section{Preprint version}


valuable, for instance to have an insight on complex turbulent liquid-gas flow like atomization for which there is no other available tools. However, it would be beneficial to go further in the validation process by evaluating under-resolved regions of such numerical simulation with a specific indicator.

From a physical point of view the minimum size of structures encountered in a twophase system should be governed by an equilibrium between inertial forces and surface tension force. In a sense, it should be possible to define a criterion describing turbulenceinterface interactions and the smallest scale emerging from these interactions. The evaluation of this scale is of utmost importance, but there are still no studies in the literature that demonstrate theoretically the smallest scale of a turbulent liquid-gas flow system, such as the Kolmogorov scale in single-phase flow. Recently, Thiesset et al. (2020) has investigated the energy transfer between the different scales of the liquid volume fraction field based on two-point statistics, showing a possible way to find the smallest scale of a turbulent two-phase environment. Regarding sprays, minimum droplet scales are obtained based on Weber numbers that represent the stability of a droplet experiencing a shear with the surrounding gas. This approach is restricted to sprays composed by spherical droplets having no additional internal excitation. Dense liquid-gas flows have a more complex physics that include other effects like collision and liquid-gas surface interaction. Thus, the definition of the Weber number is an issue by itself, the inertial force that have to be compared with the surface tension force are mostly based on the slip velocity between the liquid and the surrounding gas for dispersed spray. Consequently, this is the gas density that is considered, but for dense spray, the collision of liquid-liquid interface is more likely. Therefore, the inertial force to be considered should be defined using the liquid density together with a characteristic velocity that is related to the liquid agitation, or even more precisely to the surface agitation. Statistically, such quantity should be characterized by the turbulent kinetic energy of the surface velocity. To complete a possible Weber number definition, in dispersed spray, the surface tension force is related to a length scale usually defined as the diameter of the droplet. In dense 
spray, the notion of spherical droplet is lost, the possible length scale to be considered is either the local curvatures or the average distance between two interfaces. From the previous work (Duret et al., 2013) a global length scale has been retained based on the surface density, but clearly it hides a complete PDF of possible curvature that can be 5 negative and positive. During these collisions, curvatures at the contact time can take nearly all-possible value. As a result, the interface geometry is much more complex than a sphere: it can be for instance a ligament or a liquid sheet. Consequently, any interface wrinkling should be considered.

From the pioneering work of Vallet and Borghi (1999) the description of this dense liquid-gas flows has been addressed through the so-called ELSA (Eulerian Lagrangian Spray Atomization) model (Anez et al. (2018); Lebas et al. (2009); Vallet and Borghi (1999); Vallet et al. (2001)). Within this approach no assumption is done on the geometry of the liquid-gas interface, but the total amount of surface per unit of volume is determined. It has been assumed that for a given amount of liquid under the influence of a fixed intensity of turbulence, the ratio between the turbulent kinetic energy and the surface energy statistically reaches an equilibrium value. This ratio is thus an equilibrium turbulent Weber number related to the characteristic length scale of any wrinkling of the interface. Since then, no theory has been developed yet to determine this value. To fill this gap, an estimation of its value has been determined through dedicated DNS of turbulent liquid-gas flows in Luret et al. (2010) and Duret et al. (2012). Due to the lack of theoretical rules to set the appropriate mesh resolution, these DNS studies have been established thanks to a mesh convergence study with respect to the measured total area of liquid-gas surface. The turbulent liquid-gas flow considered has been a statistically two-phase Homogeneous Isotropic Turbulence (HIT) in a periodic box. This relatively simple configuration (compared to a real injection configuration) has allowed the mesh resolution to be refined until the mesh convergence has been achieved for essential characteristics such as mass and total amount of surface. But this effort is generally challenging or unfeasible on most configurations, illustrating the need to build a useful

\section{Preprint version}


indicator that can be computed on the fly to evaluate the quality of the resolution.

Recently, Anez et al. (2018) used the ELSA approach in the context of atomization LES. In this work, a coupling between numerical methods dedicated to resolved interface and sub-grid interface method has been achieved. To determine which method is

5 the most suitable during the computation, the notion of a Interface Resolution Quality (IRQ) sensor has been proposed. The first proposed IRQ sensor is based on the surface density model that determine if the total amount of surface estimated can be solved with the local grid resolution. This IRQ sensor rely on a model that include an estimation of the sub-grid amount of surface and thus not applicable for DNS simulation. But others IRQs sensor, focusing on the resolution of the interface geometry, can be relevant for DNS with resolved interface.

In this work, we propose to categorized resolved interface methods by three categories: Diffuse Interface (DI) method (for instance Saurel and Pantano (2018)) where the interface thickness is bigger than the mesh resolution, Interface Capturing (IC) approach where the interface is supposed to be infinitely thin with respect to the grid resolution but reconstruction of the interface at subgrid level is avoided (for instance the interFOAM solver Deshpande et al. (2012)) and the last category, Interface Capturing method with a Sharp Interface formalism (ICSI) where the concept of sharp interface is maintain either explicitly by a geometrical method (for instance VOF-PLIC Gueyffier et al. (1999)) or implicitly through the distance function (Sussman et al. (1994)). Beware that this classification is subject to caution and can lead to further discussions among specialists but is necessary to have a better understanding on the definition and implementation of the $I R Q$ in various context. The last category is accepted to be the most accurate one, since this kind of formalism allow to apply the jump condition across the interface in the most accurate way.

Wardle and Weller (2013) proposed an indicator that is related to IRQ, based on the gradient of the liquid volume fraction $\alpha$. In their numerical method, the transition between liquid to gas phase through the interface should be achieved in less than one 
cell. Accordingly, the gradient of the liquid volume fraction should be greater than $\frac{1}{\Delta x}$. Whenever the gradient is less than this value, numerical diffusion have damped the transition between phases. This indicator highlights the eventual difficulty of such methods to represent a sharp transition in a poorly-resolved scenario. Moreover, such criterion is 5 useful to detect numerical diffusion if the resolved interface method included a defined length scale for the phase transition, which can be the case of certain DI and IC method. However, the last category of resolved interface (ICSI method) cannot experience any numerical diffusion of the phase indicator, since the profile is assumed to be a step profile across the interface by definition. In such method, the geometry of the interface itself has to be evaluated. The proposed indicator Anez et al. (2018) simply compares the total curvature to the mesh resolution. The curvature is a general output expected from all numerical formalism, since it is necessary to calculate surface tension forces. Therefore, the curvature-based IRQ can be used to evaluate the level of accuracy of the interface resolution for many kind of numerical method.

To illustrate this indicator potential, the well-known ARCHER code (Canu et al. (2018); Duret et al. (2018, 2013); Ménard et al. (2007); Vaudor et al. (2017)) is used first on the two phase HIT configuration, where a proper mesh convergence can be achieved. This configuration is designed to mimic a turbulent liquid-gas flow relevant for application such as injection and atomization. The two-phase HIT numerical simulation is presented together with the main numerical feature of the ICSI method used in this work. Then, a mesh convergence study is conducted to understand the effect of low resolution on a chosen output of the computation. The curvature-based indicator, $I R Q_{\kappa}$ is then defined and analyzed statistically. An attempt is done to summarize the information than can be extracted from the $I R Q_{\kappa}$ to have a first a posteriori estimation of the level of resolution obtained in a given liquid-gas flow numerical simulation.

Finally, a more realistic configuration corresponding to the Spray A from the ECN (Engine Combustion Network) is studied. It is evident that the real Weber and Reynolds number are too high for the flow to be described by the DNS formalism. However, it is a

\section{Preprint version}


good candidate to observe the behavior of the IRQ indicator for different range of Weber and Reynolds number. To achieve this, Weber and Reynolds numbers are reduced to reach their usual order of magnitude encountered in most liquid jet DNS studies.

\section{GOVERNING EQUATIONS AND NUMERICAL METHODS}

\section{$5 \quad 2.1$ Navier-Stokes equations and interface capturing method}

Here, a joint level set/VOF method are coupled with a projection method to carry out the direct numerical simulation of incompressible Navier-Stokes equations:

$$
\frac{\partial \vec{V}}{\partial t}+(\vec{V} \cdot \nabla) \vec{V}=-\frac{\nabla p}{\rho}+\frac{1}{\rho} \nabla \cdot(2 \mu \vec{D})+\vec{f}+\frac{1}{\rho} \sigma H \delta(\varphi) \vec{n}
$$

where $p$ is the fluid pressure, $\vec{V}$ the velocity vector, and $\vec{D}$ the viscous deformation tensor, $\rho$ the density, $\mu$ is the dynamic viscosity, $\sigma$ the surface tension and $H$ the mean curvature. At the interface, the surface tension force can be considered based on the Dirac function $\delta(\varphi)$, where $\varphi$ is the Level-Set function. Concerning the interface capturing method, a Coupled Level Set Volume Of Fluid (CLSVOF) interface capturing method is used to ensure mass conservation and a accurate description of the interface topology (See Ménard et al. (2007) for details).

This work benefits for the latest improvements of the ARCHER code : the convective term is solved using the improved Rudman technique presented in Vaudor et al. (2017), allowing a better accuracy and description of high density ratio flows. This method is based on the computation of mass fluxes from the VOF method, which can then be used in a conservative formalism of the convective term. The diffusive term is computed with the approach of Sussman et al. (2007), the main advantage of this method is the implicit jump condition of the viscous tensor. For further details, a review of the numerical methods dedicated to the computation of the viscous term in two-phase incompressible flows is available in Lalanne et al. (2015).

Fluid dynamics equations are solved in the context of a low Mach number approach, 
based on a projection method for the direct numerical simulation of incompressible Navier-Stokes equations (detailed in Vaudor et al. (2017)). The viscosity depend on the sign of the level set function according to each phase (liquid and gas). To finalize the description of the two-phase flow, jump conditions across the interface are taken into 5 account with the ghost fluid (GF) method (Kang et al. (2000); Liu et al. (2000)).

The evaluation of the curvature is based on the distance function $(\varphi)$ that is part of the numerical procedure in the resolution of the two-phase flow Navier-Stokes equations.

The normal to the surface $\vec{n}=-\frac{\vec{\nabla} \varphi}{|\vec{\nabla} \varphi|}$ is first calculated. Then, the matrix $P=I-\vec{n} \vec{n}^{T}$ with $I$ the identity matrix, and the Hessian matrix $H_{m}=\frac{\partial^{2} \varphi}{\partial x_{i} \partial x_{j}}$ are calculated in order 10 to have the matrix $G_{m}=-\frac{P H_{m} P}{|\vec{\nabla} \varphi|}$. Finally, the two principal curvatures $\kappa_{1}$ and $\kappa_{2}$ are obtained as $\kappa_{1}=\frac{T+\sqrt[2]{2 F^{2}-T^{2}}}{2}$ and $\kappa_{2}=\frac{T-\sqrt[2]{2 F^{2}-T^{2}}}{2}$ with $T$, the trace of the matrix $G_{m}$ and $F$, the Frobenius norm of the matrix $G_{m}$. The reader interested in a more detailed explanation of such method is addressed to Canu et al. (2018); Kindlmann et al. (2003). Concerning the evaluation of the surface density, the surface of each cell is extracted from the Piecewise Linear Interface Calculation (PLIC) reconstruction algorithm, see Ménard et al. (2007) for details.

\subsection{Numerical configuration}

In order to study the mesh resolution effect on a turbulent liquid-gas flow the numerical configuration developed by Luret et al. (2010) and Duret et al. (2012) is retained. It correspond to a Homogeneous Isotropic Turbulence (HIT) including a liquid-gas flow with a sustained turbulence intensity induced by a linear forcing. The advantage of this configuration is the possibility to study the evolution of the flow and in particular its statistics by accumulating data both in time and space. The numerical domain is a cubic box with periodic boundaries. The physical parameters are reported on Table 1, where $\bar{k}$ the averaged kinetic energy in the whole domain and $L$ the box size. This simple configuration is supposed to be somehow representative of liquid-gas flows that may be encountered

\section{Preprint version}


in actual application such injection and atomization for instance. It has been used successfully to determine some characteristics of such flows (Bouali et al. (2016); Duret et al. $(2012,2013))$.

The same HIT configuration with only the gas phase has been presented and validated in Duret et al. (2012) where the estimation of the Kolmogorov scale, turbulence spectrum and Reynolds based on the Taylor scale has been given. This ensure that the equivalent single phase flow HIT configuration is accurately resolved before considering a two-phase flow HIT with the same turbulence parameters.

The Figure 1 represents a typical snapshot of the surface for two mean liquid volume fractions for two resolutions.

TABLE 1: Physical parameters for the HIT configuration.

\begin{tabular}{ccccccc}
\hline $\begin{array}{c}\rho_{g} \\
\left(k g \cdot m^{-3}\right)\end{array}$ & $\begin{array}{c}\rho_{l} \\
\left(k g \cdot m^{-3}\right)\end{array}$ & $\begin{array}{c}\mu_{g} \\
\left(k g \cdot m^{-1} \cdot s^{-1}\right)\end{array}$ & $\begin{array}{c}\mu_{l} \\
\left.k g \cdot m^{-1} \cdot s^{-1}\right)\end{array}$ & $\begin{array}{c}\sigma \\
N\end{array} m^{-1}$ & $\begin{array}{c}\bar{k} \\
\left(m^{2} \cdot s^{-2}\right)\end{array}$ & $\begin{array}{c}L \\
(m)\end{array}$ \\
\hline 25 & 753 & $1.879 \times 10^{-5}$ & $5.65 \times 10^{-4}$ & 0.0135 & 3.6 & $1.5 \times 10^{-4}$ \\
\hline
\end{tabular}

This figure illustrates the effect on the flow of the mean liquid volume fraction, droplets are present in majority only for very diluted case. Larger volume fractions have been studied previously where there is no droplet at all and even bubble for mean liquid volume fraction greater than $50 \%$. Following the flow in time, collisions play a very important role on the evolution of the surface characteristics. Regarding the mesh resolution, it is clear that increasing the mesh resolution allows for smallest structures to be captured. Of course the question arises: How much the mesh resolution has to be increase? Answering this question and finding a way to have an indication of the resolution quality is the topic of the following section. 

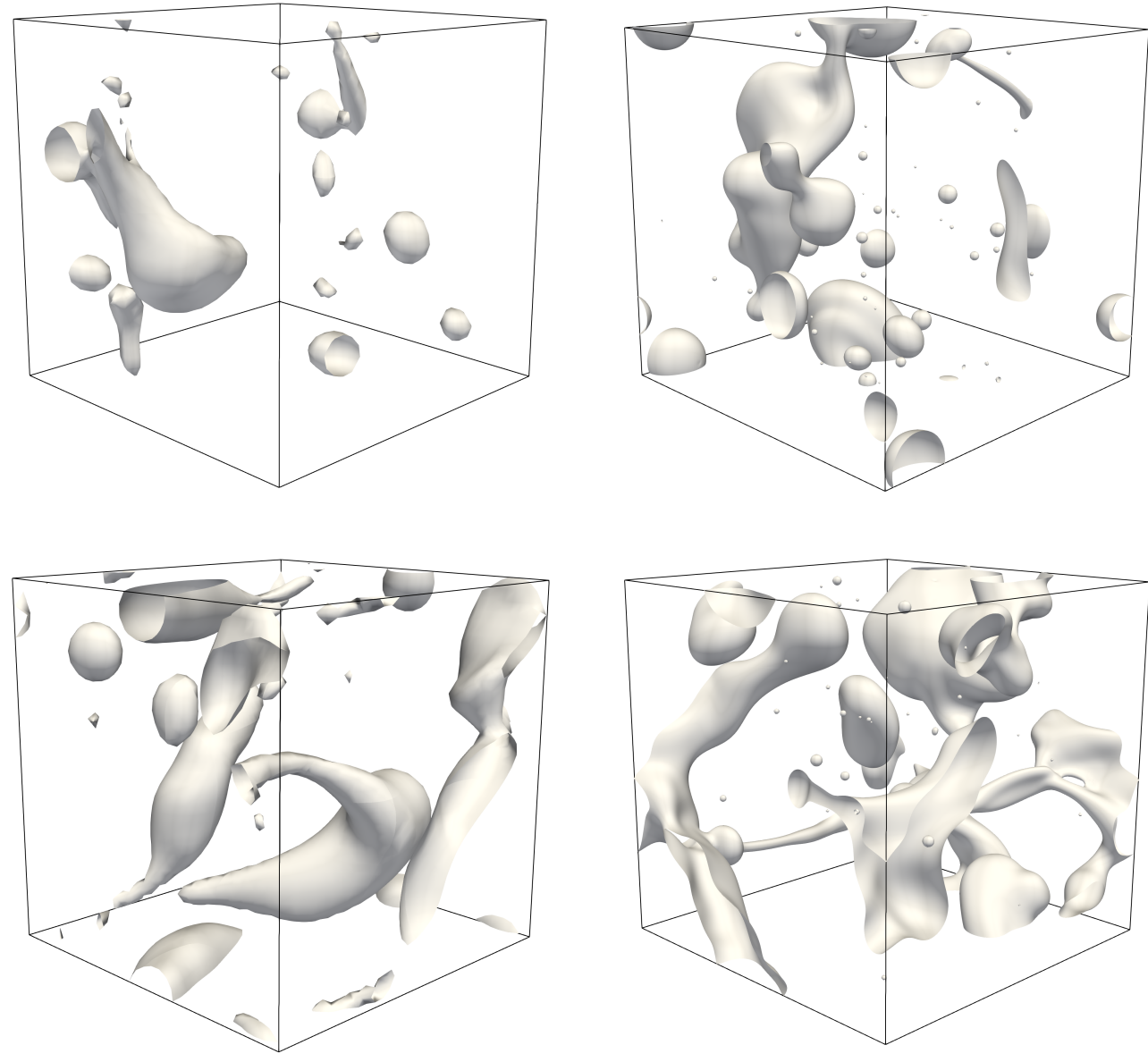

FIG. 1: Surface representation of the HIT configuration with $\alpha=5 \%$ (top) and $\alpha=10 \%$ (bottom) for a $32^{3}$ (left) and a $256^{3}$ (right) mesh.

\section{HOW TO DEFINE A WELL RESOLVED INTERFACE IN TURBULENT LIQUID GAS FLOWS?}

In turbulent liquid-gas flows, there is no equivalent theoretical definition of the smallest length scale of the flow such as the Kolmogorov scale for single phase flow. The Batchelor length scale for a scalar, such as the liquid volume fraction, is not applicable since liquid and gas are immiscible and thus the Schmidt number is infinite. Some attempts have been done to define a stable diameter of droplet experiencing a particular force : the mean shear for a droplet with a fixed slip velocity with respect to the gas phase,

\section{Preprint version}


or a droplet stressed by an homogeneous turbulence in the gas phase (Hinze (1955)). However, these approaches are valid only for specifics cases and the characteristic size of the smallest wrinkling of the interface based on a general theory of turbulence in liquid-gas flow is still missing.

Accordingly, regarding the capture of this particular scale in numerical simulation, up to now, only a mesh convergence study can be done to ensure that the flow is fully resolved. At this level it is important to acknowledge that this convergence study has to be performed on some parameters that we want to preserve. Indeed, the smallest length scale of the interface wrinkling produced by the flow tend to zero even for a simple event like the breakup of a cylindrical ligament. At the location of the breakup the cylinder is pinched down to a size that is related to the interface thickness itself. By construction the interface resolution method assumed this scale to be small compared to the mesh resolution. Thus this small event cannot be fully captured, and a breakup event will occur numerically at a scale related to the mesh resolution. Despite this drawback, numerous successes have been achieved proving that numerical simulations are able to determine for instance the size of the droplet issued of a Rayleigh-Plateau instability. Thus, although the numerical simulation is not able to capture all scales of the interface, it can be refined enough to determine some specified features or main statistics of the flow. These preserved features of the turbulent liquid-gas flow define the good resolution of the interface.

\subsection{Mass conservation and interface area}

Which features of the flow can we preserve in this turbulent liquid-gas flow? The first one should be the mass conservation, and more precisely the mass conservation by phase, so the amount of liquid and the amount of gas. The total relative mass error defined as

$25 m_{e r r}=\left|\frac{m(t)-m_{0}}{m_{0}}\right|$, where $m_{0}$ is the initial total mass and $m(t)$ the total mass temporal evolution. The temporal evolution and the maximum of the relative error are presented in Figure 2. A first order convergence is observed on the right figure with maximum 
errors going from $1.2 \%$ for a $32^{3}$ mesh to $0.2 \%$ for the $256^{3}$ mesh and this for simulations with at least ten turbulent time scales $\tau_{t}\left(\tau_{t}=\frac{\bar{k}}{\bar{\epsilon}}\right.$, where $\bar{k}$ is the mean turbulent kinetic energy and $\bar{\epsilon}$ the mean turbulent dissipation). Note that it is possible to conserve mass to machine precision in the simulation through the VOF method. However, in many case

5 it is not suitable to artificially maintain very small structures in the simulation, which in a real physical scenario may breakup under the mesh size. This will act as a numerical surface tension force that keep the liquid structure at a certain scale, related to the mesh size. To avoid this kind of issue, in our code we choose to discard them when the numerical method is not able to describe the structure. This is the source of the mass losses observed in Figure 2 .
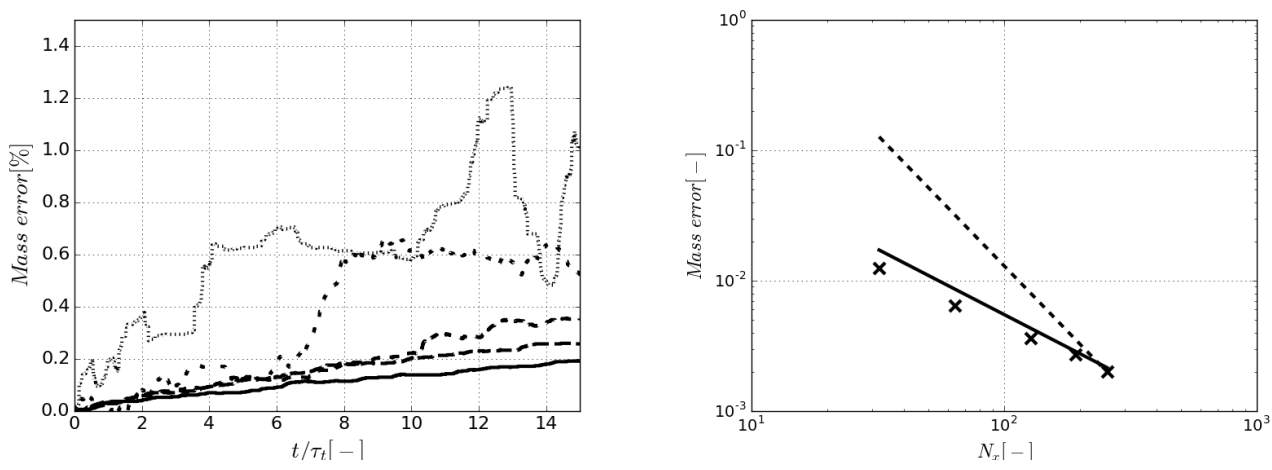

FIG. 2: Temporal evolution of the mass error (left) for the HIT configuration with $\alpha=10 \%$ for different meshes. …..., $32 \times 32 \times 32$; - - - , $64 \times 64 \times 64$; - - , $128 \times 128 \times 128$; - - - $192 \times 192 \times 192$; ,$- 256 \times 256 \times 256$. The order of convergence of the maximum mass error is showed on the right (the solid line corresponds to a first order and the dashed line corresponds to a second order).

In physical processes such as atomization and evaporation, a key quantity is the total amount of the interface area : it greatly influence the vaporization rate and the occurence of breakup and coalescence. In the literature, many modeling formalism used the transport equation of the surface density which is defined as the ratio between the

15 surface area and the volume of a given cell (for instance Anez et al. (2018); Gorokhovski and Herrmann (2008); Lebas et al. (2009); Vallet and Borghi (1999); Vallet et al. (2001)). This quantity is not a constant during the simulation due to coalescence, breakup and collision events that will have an impact on the amount of surface. But, for a certain

\section{Preprint version}


intensity of turbulence with a given proportion of liquid and gas volume, the total amount of surface should converge statistically to a mean value. From previous Figure 1, it is clear that increasing the mesh resolution leads to an increase of the amount of surface. The Figure 3 shows the variation of the amount of volume averaged surface density $\bar{\Sigma}$, 5 where $\Sigma=\frac{S}{V_{\text {cell }}}$ in a given cell, as a function of the mesh resolution. In this HIT simulation of liquid-gas flow it is possible to achieve this mesh convergence procedure since the total amount of surface becomes independent of the mesh resolution for a total mesh number per direction greater than 128. It means that at some level of resolution, even if a finer mesh resolution is supposed to slightly increase the amount of surface when

10 a breakup occurs, these events are rare enough to have any sensible effect over the whole amount of surface.

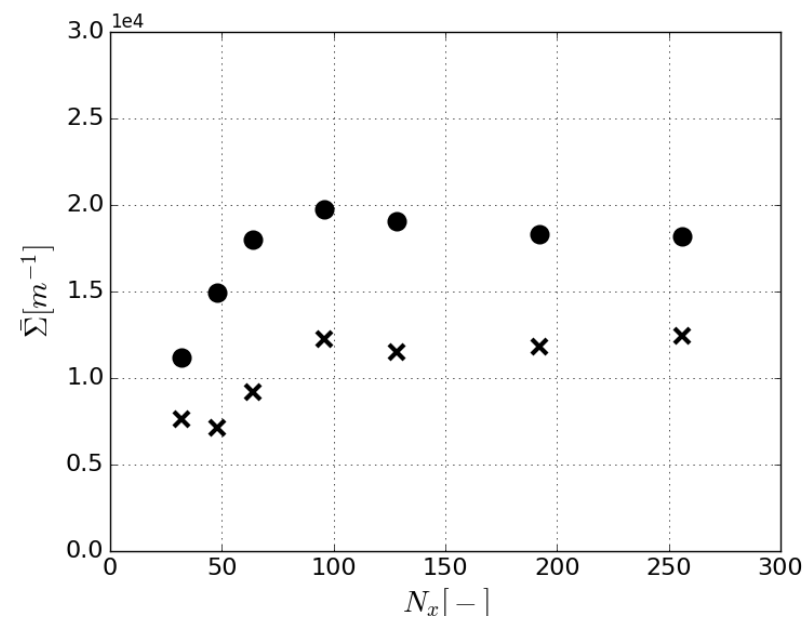

FIG. 3: Mesh convergence of the mean surface density $\bar{\Sigma}$ for the HIT configuration with $\alpha=5 \%$ (cross) and $\alpha=10 \%$ (circle).

\subsection{Impact on spray distribution and curvature}

Finally, to explore the small scales created during an atomization process, one would consider the spray size distribution. This quantity cannot be determined directly in a general turbulent liquid-gas flow since the liquid phase is not composed only by droplets 
with a given diameter. As proposed in Canu et al. (2018), this droplet size distribution can be generalized by a surface curvature distribution. For an atomization process, this function will recover the droplet size distribution at the final stage of the process but can be used meanwhile to describe surface scale distribution. The cumulative of this

5 function and its PDF are drawn on Figure 4 and Figure 5.

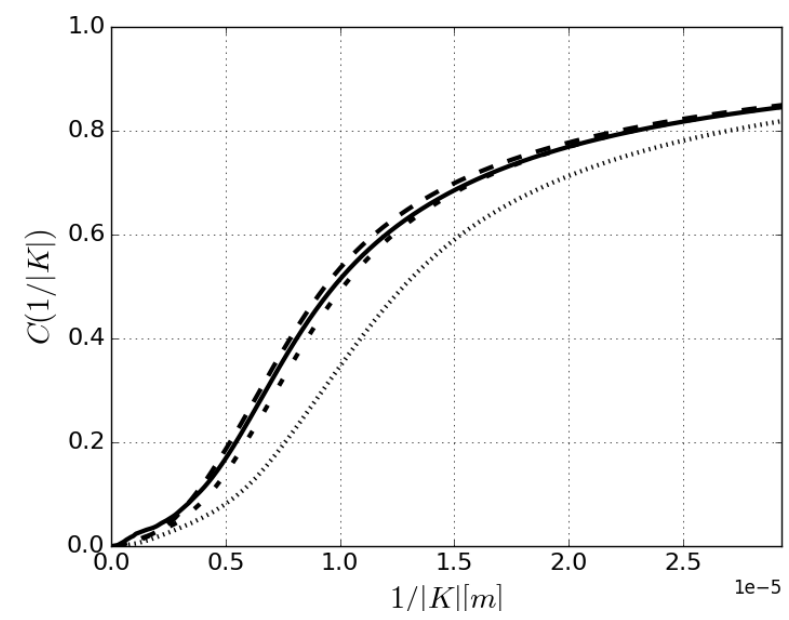

FIG. 4: Cumulative distribution function of $1 /\left|\kappa_{1}+\kappa_{2}\right|$ for the HIT configuration with $\alpha=10 \%$ for different meshes. …..., $32 \times 32 \times 32 ;-\cdots, 64 \times 64 \times 64 ;--, 128 \times 128 \times 128 ;-, 256 \times 256 \times 256$.

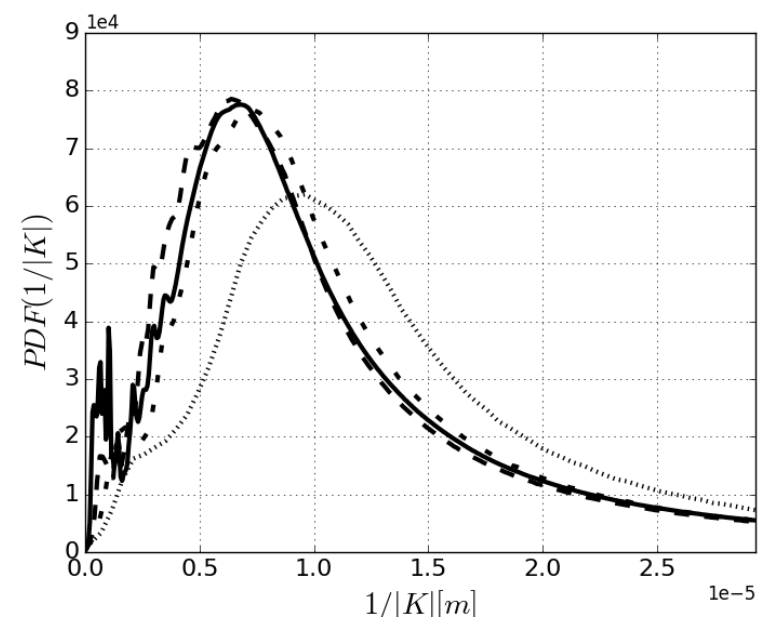

FIG. 5: PDF of $1 /\left|\kappa_{1}+\kappa_{2}\right|$ for the HIT configuration with $\alpha=10 \%$ for different meshes. ........, $32 \times 32 \times 32 ;-\cdots, 64 \times 64 \times 64 ;--, 128 \times 128 \times 128 ;-, 256 \times 256 \times 256$.

\section{Preprint version}


It appears from the Figure 4 that it is possible to refine enough the mesh in order that all curves collapse for meshes of $128 \times 128 \times 128$ and above. This corresponds also to the converged total amount of surface presented on Figure 3, showing that indeed the curvature distribution is the key parameter to capture the total amount of surface. The

5 Figure 5 shows the probability as a function of each the curvature level. Since the abscissa is one over the curvature, it represents the mean radius of curvature. The left part of the curve represents the small radius of curvature and it is clear that the convergence is not totally achieved in this zone. Part of the problem is the statistical convergence, the less you have surface the more you have to wait to establish the statistical convergence. But this observation correspond also to the problem previously described: the mesh resolution is insufficient during some breakup events since the smallest length scale tends to the interface thickness. But since the convergence is achieved on the total amount of surface, it appears that for this global quantity the more important feature of the curvature distribution to be captured is the cumulative distribution. Also a global convergence of the total surface occurs when the peak of the curvature distribution is also well captured. In this academic configuration, performing a mesh convergence study is feasible. However, in more realistic simulation like turbulent injection simulation, the computational cost is too important for such verification to be done. Thus, the next section will explore the possibility to develop a surface located indicator that can be used in realistic simulation to have an approximation of the level of resolution of the interface.

\section{DEFINITION OF AN INTERFACE RESOLUTION QUALITY (IRQ) INDICATOR}

\subsection{Previous IRQ definition}

From previous work in LES, it has been suggested to compare the resolved interface surface density to the total one that is the sum of the resolved interface plus the unresolved surface wrinkling occurring at subgrid level (Anez et al. (2018)). This local IRQ 
is not applicable here since, in one hand we are looking for a completely resolved interface, and in the other hand no equation is available in the present simulation to estimate the unresolved interface density.

Another IRQ has been proposed by Wardle and Weller (2013) based on the gradient

5 of the liquid volume fraction in the context of the solver interFoam of the library OpenFOAM®(Weller et al. (1998)). This solver contains a numerical procedure to sharpen the gradient of the liquid volume fraction $\alpha$ and to sustain a step profile across the interface position. This procedure is known to fail at sustaining the sharp transition for low mesh resolution. In this case, numerical diffusion occurs, leading to a decrease of the gradient of the volume fraction across the interface. Since the transition is supposed to occur on one mesh cell of thickness $\Delta x$, the expected gradient should be of the order of $1 / \Delta x$. Thus an IRQ criterion based on $\alpha: I R Q_{\alpha}=|\nabla \alpha| \Delta x$ is certainly relevant for this approach. It can be generalized to any diffuse interface approaches, where the length of the transition (the artificial interface thickness) $\lambda$ should appear instead of the previous $\Delta x$ thus leading to: $I R Q_{\alpha}=|\nabla \alpha| \lambda$.

However, for interface capturing method with interface reconstruction the thickness of the interface is by definition infinitely thin. During the reconstruction step, the liquid volume fraction is imposed to be a step profile across the interface. Hence, for this approach the $I R Q_{\alpha}$ is not applicable.

\subsection{A resolution indicator based on curvature: $I R Q_{\mathrm{k}}$}

One can argue that the scale of interface wrinkling is directly related to the interface curvature. When the curvature is too big, the interface reconstruction method cannot accurately represent the topological change and this event can have a local or global impact on the atomization mechanism (See Figure 1). To identify this kind of issue, a definition of the curvature IRQ: $I R Q_{\mathrm{K}}$ is proposed in this work. The $I R Q_{\mathrm{K}}$ is directly dependent of the local principal curvatures $\left(\kappa_{1}, \kappa_{2}\right)$ and the mesh size $\Delta x: I R Q_{\kappa}=\frac{1}{\Delta x\left|\kappa_{1}+\kappa_{2}\right|}$. An other definition of this criterion could be $I R Q_{\kappa}^{\max }=\frac{1}{2 \Delta x \max \left(\left|\mathrm{K}_{1}\right|,\left|\mathrm{K}_{2}\right|\right)}$; the factor 2 before

\section{Preprint version}


$\Delta x$ allowing a comparison with the first definition. Indeed, this definition is more restrictive and allows to ensure the resolution is both directions. A comparison between the two definitions is shown on Figure 6. On this figure, a ligament is considered unresolved in the second case because only one of the two main directions is well resolved. In the

5 first case, the high curvature in the first direction is compensate by the low curvature in the second direction leading to a "well" resolved ligament. In the following, the first definition $I R Q_{\kappa}=\frac{1}{\Delta x\left|\kappa_{1}+\kappa_{2}\right|}$ is nevertheless conserved in order to have a definition applicable in the majority of the computational codes. Indeed, the total or mean curvature $\kappa_{1}+\kappa_{2}$ is often affordable but not each curvature taken separately.

Note that various definition of IRQ can be used. However, only the IRQ based on curvature is "universal" since the evaluation of the mean curvature is mandatory to compute the surface tension force term in the two-phase Navier-Stokes equations. As such, we propose in the paper to study the one that can be used with most numerical methods/formalisms.
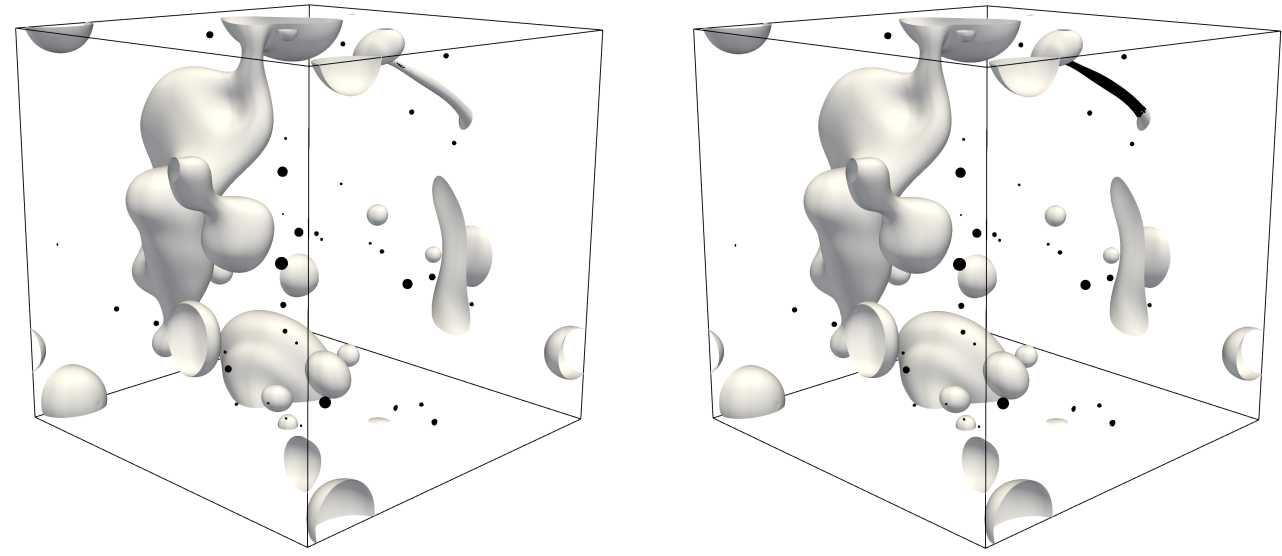

FIG. 6: Surface representation (Level Set 0 isocontour) of the HIT configuration with $\alpha=5 \%$ for $I R Q_{\kappa}=\frac{1}{\Delta x\left|\kappa_{1}+\kappa_{2}\right|}$ (left) and $I R Q_{\kappa}^{\max }=\frac{1}{2 \Delta x \max \left(\left|\mathrm{\kappa}_{1}\right|,\left|\mathrm{\kappa}_{2}\right|\right)}$ (right). The surfaces in black have an IRQ smaller than 2 .

This indicator highlights under-resolved region of the interface and, when averaged, gives an indication of the quality of the total simulation. As illustrated on Figure 7, an $I R Q_{\mathrm{K}}$ equal to 1 is equivalent to 2 mesh cells in the radius of a sphere, and consequently 
can be considered as a poorly-resolved area whereas an $I R Q_{\mathrm{K}}$ equal to 2 is equivalent to 4 mesh cells in the radius and consequently represents with a better accuracy this structure. We want to emphasize that the threshold value of 2 and 1 chosen in this paper is quite strict and has been chosen for a two-phase flow that present a wide range

5 of scales (HIT), the smallest one being droplets. However, the choice of the threshold value is arbitrary and vary depending on the numerical configuration studied and the desired accuracy of the simulation. For instance, if one is interested by the large scale instabilities created on a liquid jet for example, the threshold will be lower and is linked by definition to the mean curvature associated to this particular scale. Consequently, the threshold value used in this work is necessarily not universal and could be adapted for other configuration.

It will therefore be necessary for the user of the indicator to adapt it to its configuration and expectations. Of course, defining such an indicator may seem obvious, however the study of the statistics resulting from the analysis of this simple indicator gives valuable information on the quality of the simulation performed. We propose here both the definition of this indicator and the development of a working procedure based on statistics of IRQ. In the next section, the workflow along with qualitative and quantitative results obtained from the IRQ indicator are described.

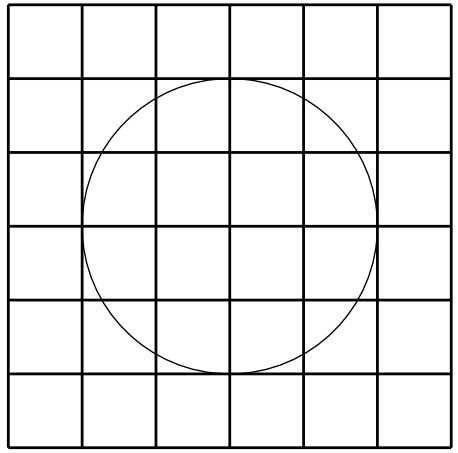

$I R Q=1$

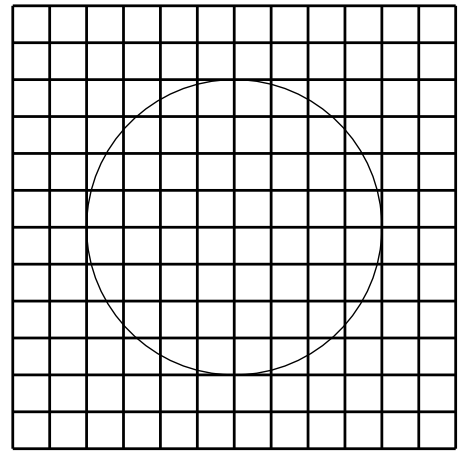

$I R Q=2$

FIG. 7: Examples of IRQ values for a sphere (2D cut plane) on different meshes.

\section{Preprint version}




\subsection{Statistics of $I R Q_{\mathrm{k}}$}

On the actual simulation the surface presented before (Figure 1) is now analyzed based on the $I R Q_{\mathrm{K}}$ (Figure 8). Using a threshold value of the $I R Q_{\mathrm{K}}$ set to 2 , the unresolved region of the surface $\left(I R Q_{\kappa}<2\right)$ are colorized in black. For the case with a low grid

5 resolution $(32 \times 32 \times 32)$ many of the unresolved scales are not captured, it is clear that the sensor $I R Q_{\kappa}$ is well adapted to identify locally the interface location that are not completely resolved. Also for the low resolution case an important percentage of the total surface is tag as unresolved ( $55 \%$ of the surface in the HIT with $5 \%$ of liquid, and $30 \%$ of the surface in the HIT with $10 \%$ of liquid which has bigger structures). On the contrary 10 for the high resolution case $(256 \times 256 \times 256)$ most of the surface is resolved and only $3 \%$ of the surface is tagged as under resolved for the HIT with $5 \%$ of liquid $(2.5 \%$ of the surface in the HIT with $10 \%$ of liquid). The given percentages are time-averaged ; indeed, the percentages fluctuate during the simulation due to the occurrence of coalescence and breakup phenomena in the turbulent configuration. Since the numerical scheme of the ARCHER code used here couple Level Set and VOF approaches in order to ensure mass preservation locally, the liquid mass is preserved even on this low resolution zone. As a result, the interface wrinkling scale is somehow kept at a scale just greater than the mesh resolution. Consequently, this numerical behaviour limits the interface deformation similarly to an additional numerical surface tension force. Other schemes certainly would have behaved differently depending of the particular treatment of the under resolved interface scenario. But as far as the scheme rely on a sharp interface definition, the sensor $I R Q_{\mathrm{K}}$ would be able to detect the unresolved region of the simulation.

Like for the curvature distribution analyzed previously, the statistic of the $I R Q_{\mathrm{K}}$ can be scrutinized to estimate the level of resolution of the interface globally. On the cumulative of IRQ (Figure 9), the percentages of unresolved surfaces observed in the previous section are retrieved. The low accuracy of the $32 \times 32 \times 32$ case is visible with an abrupt slope for the low values of $I R Q_{\kappa}$. This profile is more and more spread as the mesh is refined. As expected, a very low percentage of unresolved surfaces is observed for the 

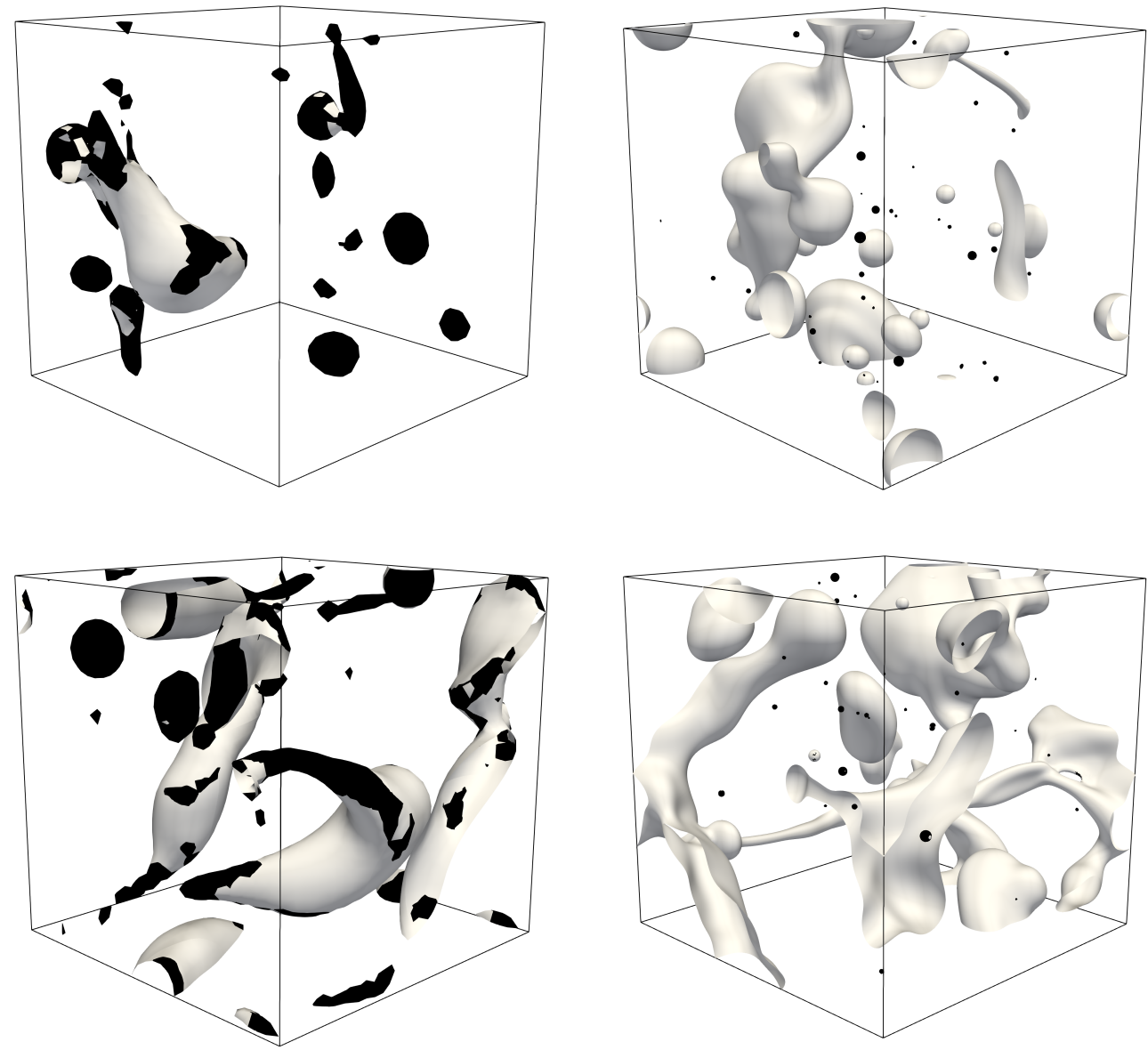

FIG. 8: Surface representation (Level Set 0 isocontour) of the HIT configuration (Level Set 0 isocontour) with $\alpha=5 \%$ (top) and $\alpha=10 \%$ (bottom) for a $32^{3}$ (left) and a $256^{3}$ (right) mesh. The surfaces in black have an $I R Q_{\kappa}$ smaller than 2 .

finest mesh $256 \times 256 \times 256$.

The PDF of $I R Q_{\kappa}$ (Figure 10) gives an useful insight of the simulation quality, based on the probability peak position. For the $32 \times 32 \times 32$ case, the peak is in the low resolution zone. As in the convergence study of the surface density (Figure 3), the simulation can

5 be considered as well resolved with meshes with more than $128 \times 128 \times 128$ cells. For the $64 \times 64 \times 64$ case, even if the peak is not in the hatched zone, a non negligible part of the curve is still inside, showing that this case can not be considered well resolved. Again, the statistical convergence is not established in the low $I R Q_{\mathrm{K}}$ zone for the same

\section{Preprint version}


reasons than for the PDF of curvature.
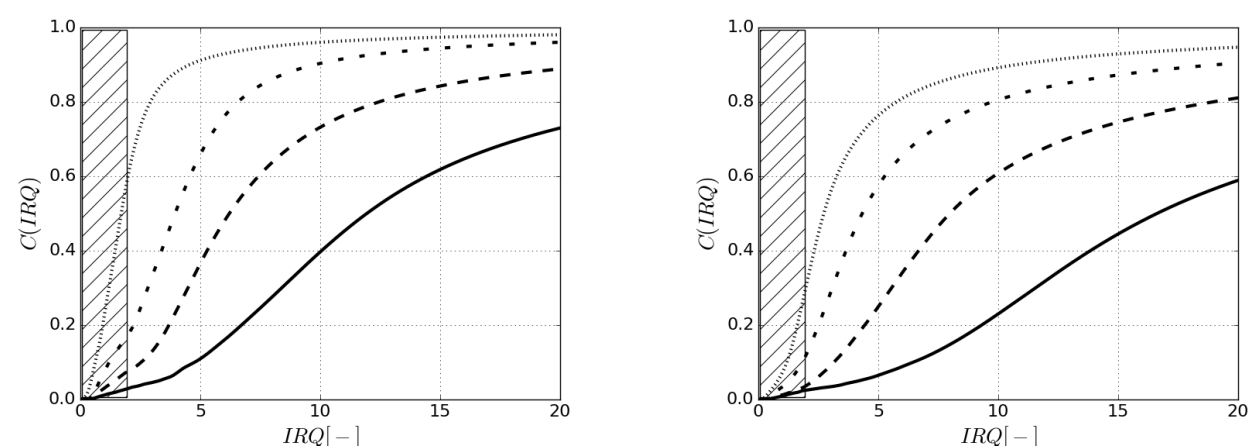

FIG. 9: Cumulative distribution function of $I R Q_{\mathrm{K}}$ for the HIT configuration with $\alpha=5 \%$ (left) and $\alpha=10 \%$ (right) for different meshes. ......, $32 \times 32 \times 32 ;-\cdots, 64 \times 64 \times 64$; - - , $128 \times 128 \times 128$; ,$- 256 \times 256 \times 256$. The hatched zone corresponds to an IRQ less than 2 .
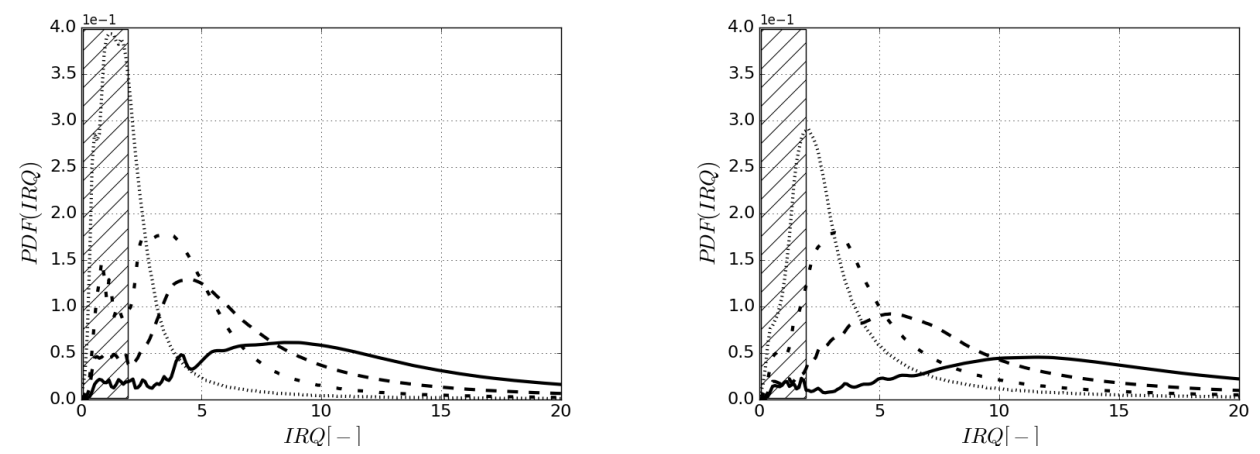

FIG. 10: PDF of $I R Q_{\kappa}$ for the HIT configuration with $\alpha=5 \%$ (left) and $\alpha=10 \%$ (right) for different meshes. …..., $32 \times 32 \times 32 ;-\cdots, 64 \times 64 \times 64 ;--, 128 \times 128 \times 128 ;-, 256 \times 256 \times 256$. The hatched zone corresponds to an $I R Q_{\mathrm{K}}$ less than 2 .

\section{APPLICATION ON LIQUID JET INJECTION}

\subsection{Numerical configuration}

The second case to be studied is a diesel jet based on the ECN (Engine Combus-

5 tion Network) Spray A configuration. The domain measures $445 \times 445 \times 1780 \mu \mathrm{m}^{3}$ and the simulation is performed on a $256 \times 256 \times 1024$ grid. The injector has a diameter $D_{i n j}=89.4 \mu \mathrm{m}$. For the inlet boundary condition, the velocities are extracted from the outlet plane of a LES simulation (performed in Anez et al. (2018)) in order to capture the 
turbulent boundary layers which interact with the injector wall. All the other boundaries are outflow boundary conditions. The physical parameters are shown in Table 2 .

TABLE 2: Physical parameters for the original ECN Spray A configuration with $W e \simeq$ 1044 and $R e \simeq 70356$.

\begin{tabular}{|c|c|c|c|c|}
\hline $\begin{array}{c}\rho_{g} \\
\left(k g \cdot m^{-3}\right)\end{array}$ & $\begin{array}{c}\rho_{l} \\
\left(k g \cdot m^{-3}\right)\end{array}$ & $\begin{array}{c}\mu_{g} \\
\left(k g \cdot m^{-1} \cdot s^{-1}\right)\end{array}$ & $\left(k g \cdot m^{-1} \cdot s^{-1}\right)$ & $\begin{array}{c}\sigma \\
N \cdot m^{-1}\end{array}$ \\
\hline 22.8 & 713 & $1.8343 \times 10^{-5}$ & $7.248 \times 10^{-4}$ & 0.0243 \\
\hline
\end{tabular}

This corresponds to the original Spray A case with high Weber $(W e \simeq 1044)$ and Reynolds $(R e \simeq 70356)$ numbers. The relations used for these numbers are :

$$
W e=\frac{\rho_{g} u_{\max }^{2} D_{g}}{\sigma}
$$

5

$$
R e=\frac{\rho_{l} u_{\max } D_{i n j}}{\mu_{l}}
$$

where $u_{\max }=800 \mathrm{~m} . \mathrm{s}^{-1}$, which is the maximum velocity encountered in the simulation with realistic parameters, and $D_{g}=\Delta x$, based on the study of Ménard et al. (2007) for the Weber number estimation. All jet configurations presented in the paper have the same mesh resolution, consequently there is no variation of the Weber number due to the mesh size alone. To study different Weber number, only the surface tension value is modified.

This case can't be described accurately in DNS due to these high numbers but it can be interesting to see the overall simulation accuracy with the original parameters and to observe the behavior of our indicator in such configuration.

\subsection{Results}

Three differents couples of Weber and Reynolds number are investigated for this configuration to illustrate the IRQ indicator behavior: a realistic case with high We/Re, an

\section{Preprint version}




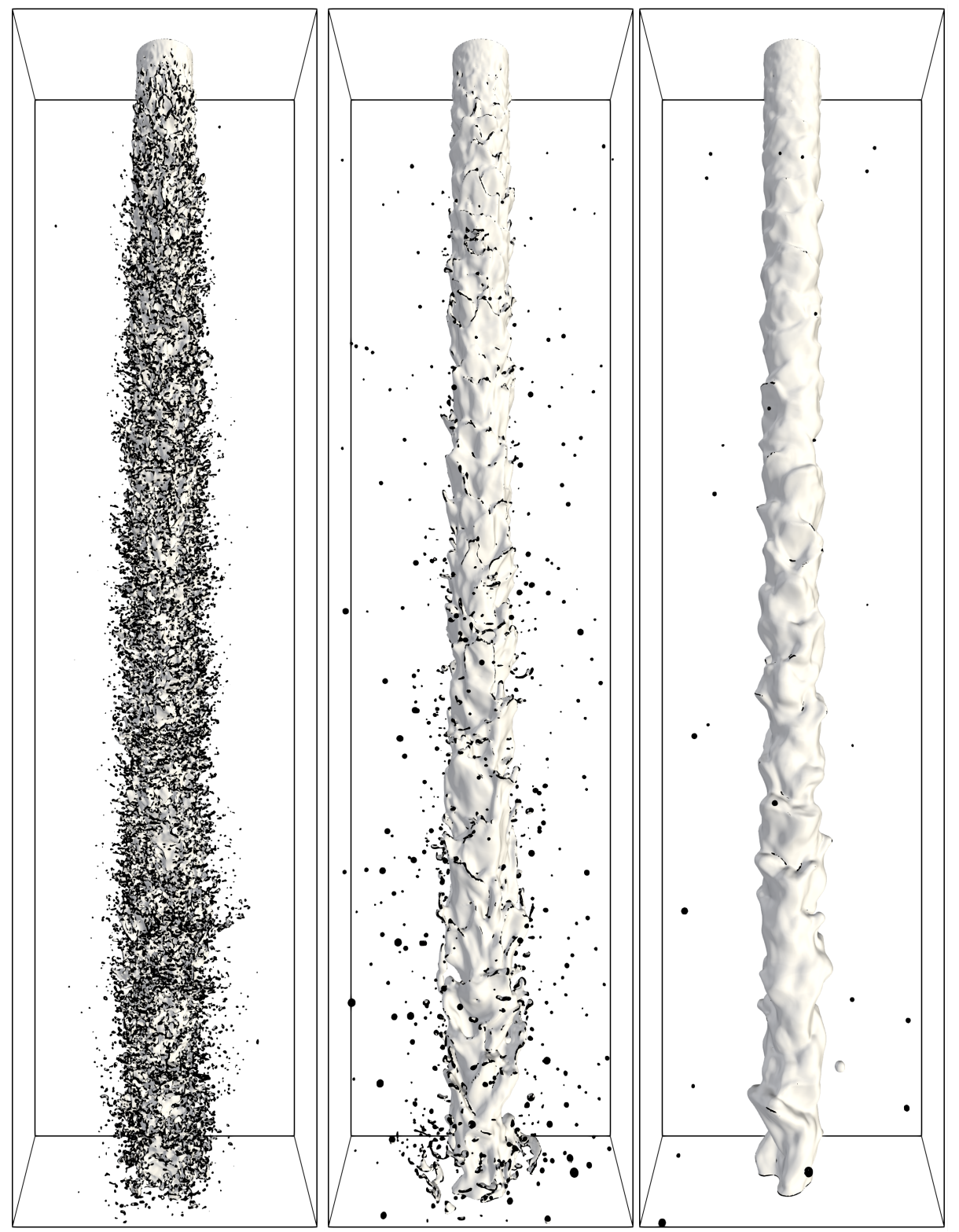

FIG. 11: Surface representation (Level Set 0 isocontour) of the Spray A configuration at $t=1.6 \times$ $10^{-5} \mathrm{~s}$. The surfaces in black have an $I R Q_{\mathrm{K}}$ smaller than 2. Left : original Spray A configuration (We $\simeq 1044$ and $R e \simeq 70356)$; center : low Weber and low Reynolds (We $\simeq 10$ and $R e \simeq 5795)$ ; right : the most resolved case (We $\simeq 2.5$ and $R e \simeq 5795)$.

intermediary We/Re numbers case and finally a low We/Re numbers case. In the following, statistics are performed once the jet has crossed the whole domain and is fully 
established, which is after $t=0.004 \mathrm{~ms}$ for the highest velocity. The same departure is kept for other cases.

Realistic configuration : High We/Re numbers

On Figure 11 (left), the inability to represent this case with this mesh resolution with a

5 DNS approach is shown. The majority of the droplets presents a low IRQ and, consequently, are poorly represented. A zoomed view is available on Figure 12 to observe closely the interface wrinkling. The surface of the central jet is very wrinkled, presenting areas with high curvature which are not well resolved.

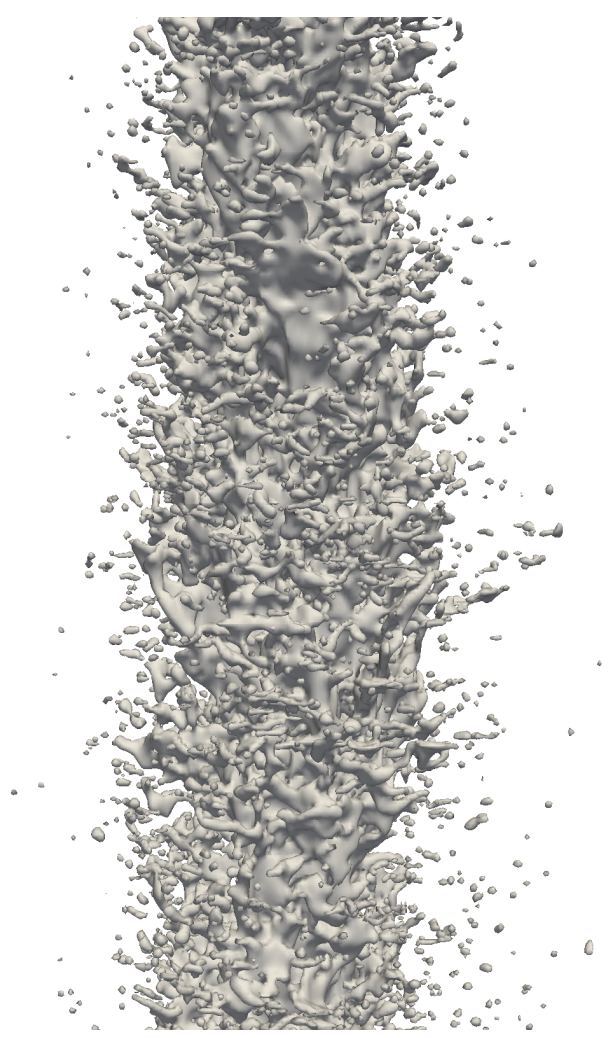

FIG. 12: Zoomed view of the original Spray A configuration case presented in Figure 11. Surface representation based on the Level Set function 0 isocontour.

The under-resolved regions represent, in this case, $33.5 \%$ of the surface $\left(I R Q_{\mathrm{K}}<1\right.$ 10 in Figure 13) and $26 \%$ of the surface is midly resolved $\left(1<I R Q_{\kappa}<2\right.$ in Figure 13). The

\section{Preprint version}


slight peak in the curves can be explained by the remaining liquid structures that broke in the wake of the mushroom head of the liquid jet, this behavior is seen in many liquid jet injection simulation (for instance Shinjo and Umemura (2010)). Consequently, some droplets are produced close to the free boundaries conditions and are then evacuated

5 by the flow. But the smaller the velocity are, the longer it takes to evacuate these kinds of liquid structure. This behavior is more present for slower Weber and Reynolds numbers.

The averaged in time PDF of $I R Q_{\mathrm{K}}$ (Figure 14 top left) confirms the previous observation about the resolution with a peak around an $I R Q_{\mathrm{\kappa}}$ value of 0.6 and a low standard deviation. Indeed, the PDF tends rapidly toward 0 for low values of $I R Q_{\mathrm{K}}$ in the resolved part. Besides, the probability tends also toward 0 when $I R Q_{\kappa}$ tends toward 0 and.$n$ accumulation of structures of a size close to $d x$ is observed. This behavior is due to the CLSVOF numerical scheme which prevents high curvatures in order to limit the situations which can lead to mass loss. As expected, we can see that for this particular range of high Weber and Reynolds numbers, performing an accurate computation is a challenging task.
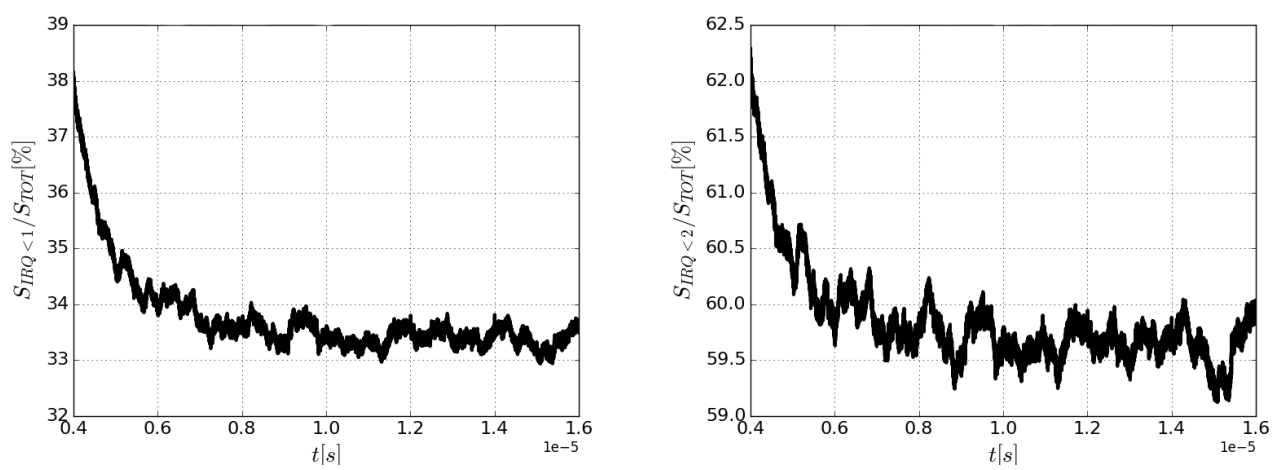

FIG. 13: Temporal evolution of the percentage of surfaces with an $I R Q_{\mathrm{K}}$ less than 1 (left) and less than 2 (right) for the original Spray A configuration (We $\simeq 1044$ and $R e \simeq 70356)$.

Moderate We/Re numbers

Most simulations of jet are performed with lower Weber and Reynolds numbers. So, another Spray A case has been performed with $W e \simeq 10$ and $R e \simeq 5795$ like in Ménard 

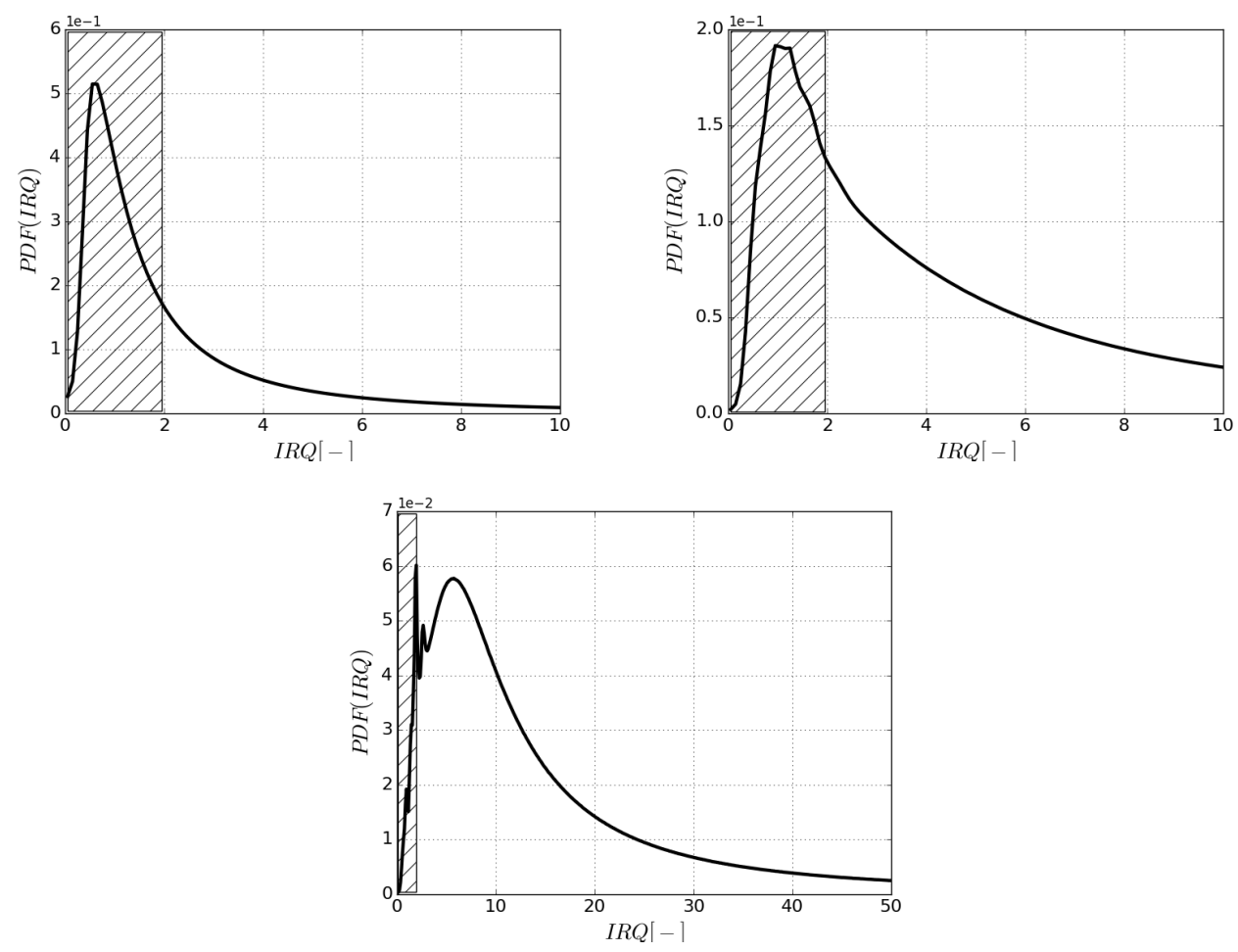

FIG. 14: PDF of $I R Q_{\mathrm{K}}$ averaged in time for the original Spray A configuration (We $\simeq 1044$ and $R e \simeq 70356$ ) (top left), the low Weber and low Reynolds (We $\simeq 10$ and $R e \simeq 5795$ ) (top right) and the most resolved case $(W e \simeq 2.5$ and $R e \simeq 5795)$ (bottom). The hatched zone corresponds to unresolved area $\left(I R Q_{\kappa}<2\right)$.

et al. (2007) in order to have a better resolution. The new parameters are in Table 3. However, the injection conditions are the same than the previous case in order to mainly capture the effect, on the interface, of fluid physical parameters which are supposed to limit its deformation.

TABLE 3: Physical parameters for the ECN Spray A configuration with $W e \simeq 10$ and $R e \simeq 5795$.

\begin{tabular}{ccccc}
\hline $\begin{array}{c}\rho_{g} \\
\left(k g \cdot m^{-3}\right)\end{array}$ & $\begin{array}{c}\rho_{l} \\
\left(k g \cdot m^{-3}\right)\end{array}$ & $\begin{array}{c}\mu_{g} \\
\left(k g \cdot m^{-1} \cdot s^{-1}\right)\end{array}$ & $\begin{array}{c}\mu_{l} \\
\left(k g \cdot m^{-1} \cdot s^{-1}\right)\end{array}$ & $\begin{array}{c}\sigma \\
N\end{array} m^{-1}$ \\
\hline 22.8 & 713 & $1.8343 \times 10^{-5}$ & $8.8 \times 10^{-3}$ & 2.54 \\
\hline
\end{tabular}
of the under-resolved surfaces comes from small droplets at the periphery of the central

\section{Preprint version}


jet. However, here, much less under-resolved areas (colorized in black for $I R Q_{\kappa}<2$ ) are visible. Due to the low level of turbulence (low Reynolds number) and the high surface tension force (low Weber number), all the instabilities with a high space frequency are damped and the developed structures have a lower curvature allowing a better res-

5 olution. The percentage of surface midly resolved is somewhat lower than the original case but the under-resolved surfaces are strongly reduced (Figure 15). It corresponds to only $8 \%$ of the total surface. The PDF of $I R Q_{\kappa}$ (Figure 14 top right) is shifted toward the higher values of $I R Q_{\mathrm{K}}$ and the peak is around 1.1. Higher values of $I R Q_{\mathrm{K}}$ and, so, lower values of curvature are more represented and the PDF profile is more smeared than the previous case. Even if this case with lower Weber and Reynolds numbers is obviously more resolved than the original case, there is still $23 \%$ of the surface that has an $I R Q_{\kappa}$ less than 2 , illustrating an accuracy not as good as expected. However, it's more difficult to be well resolved in this kind of simulation where the injected turbulence have wider ranges of scales, which are consequently atomizing more the jet, than other studies with similar parameters but where the synthetic injected turbulence is mostly composed of large scales (see Ménard et al. (2007) for instance). Indeed, here, the injected turbulence comes from a LES simulation realized inside an injector where large eddies are present in the center and small eddies are created close to the walls, in the boundary layer, disrupting the interface. Moreover, the threshold value used for $I R Q_{\kappa}$ is probably too high for this kind of application as explained in the previous section.

\section{Low We/Re numbers}

Finally, in order to see what parameters have to be chosen to have an accurate simulation of the jet, a third case with this time $W e \simeq 2.5$ and $R e \simeq 5795$ has been performed. The parameters are reported on Table 4 .

Only a small fraction of the total surface is now under-resolved (Figure 11 right). Even if the simulation is much longer to reach a steady state (Figure 16), results obtained show that the mean percentage of surface that has an $I R Q_{\kappa}$ less than 2 is around $2 \%$ and 

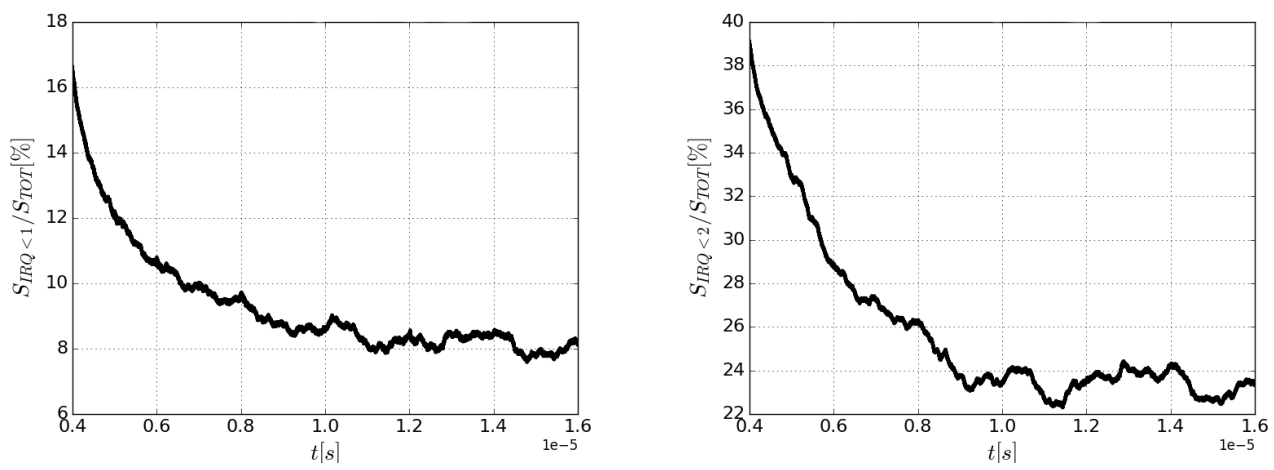

FIG. 15: Temporal evolution of the percentage of surfaces with an $I R Q_{\kappa}$ less than 1 (left) and less than 2 (right) for the Spray A configuration with a low Weber and a low Reynolds ( $W e \simeq 10$ and $R e \simeq 5795)$.

TABLE 4: Physical parameters for the ideal ECN Spray A configuration with $W e \simeq 2.5$ and $R e \simeq 5795$.

\begin{tabular}{ccccc}
\hline $\begin{array}{c}\rho_{g} \\
\left(k g \cdot m^{-3}\right)\end{array}$ & $\begin{array}{c}\rho_{l} \\
\left(k g \cdot m^{-3}\right)\end{array}$ & $\begin{array}{c}\mu_{g} \\
\left(k g \cdot m^{-1} \cdot s^{-1}\right)\end{array}$ & $\begin{array}{c}\mu_{l} \\
\left(k g \cdot m^{-1} \cdot s^{-1}\right)\end{array}$ & $\begin{array}{c}\sigma \\
N\end{array} m^{-1}$ \\
\hline 22.8 & 713 & $1.8343 \times 10^{-5}$ & $8.8 \times 10^{-3}$ & 10.16 \\
\hline
\end{tabular}
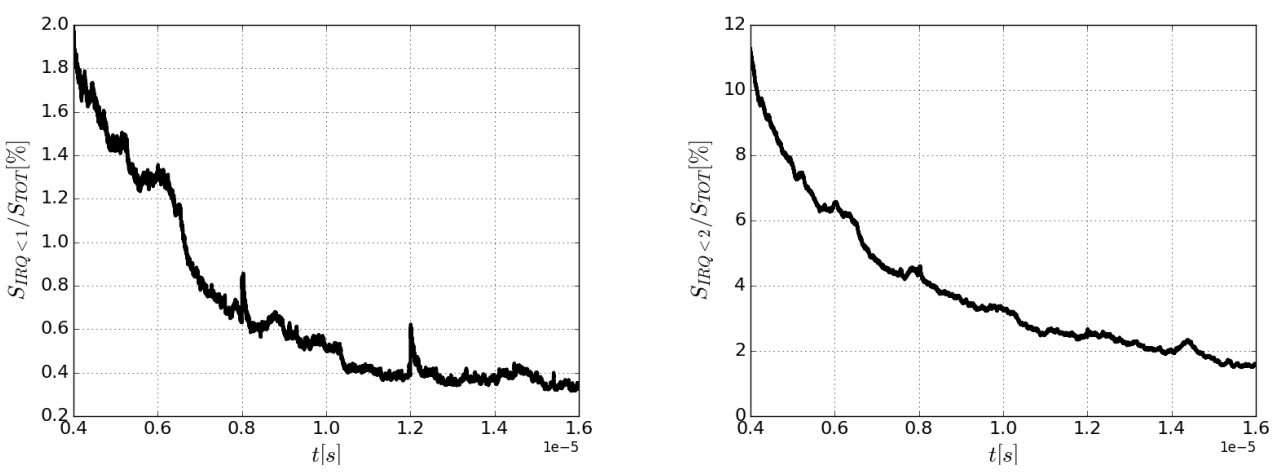

FIG. 16: Temporal evolution of the percentage of surfaces with an $I R Q_{\mathrm{K}}$ less than 1 (left) and less than 2 (right) for the most resolved Spray A configuration ( $W e \simeq 2.5$ and $R e \simeq 5795$ ).

only $0.4 \%$ of the surface is really under-resolved $(\operatorname{IRQ}<1)$. Also, the peak of the PDF of $I R Q_{\mathrm{K}}$ is now in the resolved part of the graph and is around an $I R Q_{\mathrm{\kappa}}$ value of 6 (Figure 14 bottom). This third case is obviously well resolved but to reach this level of accuracy, physical parameters far from the experimental ones have to be chosen and 5 the jet atomizes itself with a few production of droplets.

\section{Preprint version}




\section{CONCLUSION}

In this work, an estimation of a resolution indicator called $I R Q_{\mathrm{K}}$ is performed. The indicator allows to obtain a first qualitative overview of the under resolved regions of a twophase simulation. Then, through its statistics, the indicator gives a qualitative overview of the resolution of the simulation. This indicator is defined by the inverse of the product of the mesh size and the mean curvature. Consequently, this sensor can be computed in many interface capturing formalism. When the $I R Q_{\mathrm{K}}$ is lower than a threshold value, the considered region is estimated under-resolved. The chosen threshold value depend on the mean curvature of the scale of interest (i.e. droplets, bubbles, instabilities...). In this work, a threshold value of 1 and 2 has been chosen and investigated, the smallest scale being droplets. However, these threshold are not universal and should be adapted to the investigated configuration.

Two main configurations have been used to illustrate the potential of the $I R Q_{\kappa}$ : the two-phase homogeneous isotropic turbulence and the Spray A liquid jet from the ECN. The HIT configuration serves as a reference to evaluate the accuracy that can be expected on a well-resolved case. It is shown that, even for a well resolved case, there are some under-resolved regions during breakup events which can not be captured. These regions are highlighted by the $I R Q_{\kappa}$ indicator and the shape of the PDF of curvature. However, a convergence is observed for the mains statistics of the two-phase flows, illustrating that, after a certain level of mesh resolution, the contribution of underresolved regions to the statistics is negligible on the mean surface density. Then, the Spray A configuration points out, through the $I R Q_{\mathrm{k}}$ indicator statistics, the fact that a sufficient resolution is difficult to reach for realistic parameters. Same simulations with a lower Weber and Reynolds numbers are then performed to capture the main flow characteristics, which translate into attaining a satisfying level of IRQ PDF shapes. Further work will be dedicated to study the accumulation and the inflection of the IRQ PDF close to the cell size, which represent artificial liquid structure that can not break or disappear depending on the numerical method used. These structures will have an influence on the 
droplet distribution at small diameters which is frequently studied in DNS of two-phase flows.

Preprint version 


\section{ACKNOWLEDGEMENTS}

This work was granted access to the HPC resources of CRIANN (project 2006011), IDRIS, TGCC and CINES under the allocation A0052B06153 and A0072B06153 made by GENCl (Grand Equipement National de Calcul Intensif). This research was supported

5 by the Normandy region (NEPTUNE project).

\section{REFERENCES}

Anez, J., Ahmed, A., Hecht, N., Duret, B., Reveillon, J., and Demoulin, F.X., Eulerian-Lagrangian spray atomization model coupled with interface capturing method for diesel injectors, International Journal of Multiphase Flow, 2018.

Bouali, Z., Duret, B., Demoulin, F.X., and Mura, A., DNS analysis of small-scale turbulence-scalar interactions in evaporating two-phase flows, International Journal of Multiphase Flow, vol. 85, pp. 326-335, 2016.

Canu, R., Puggelli, S., Essadki, M., Duret, B., Menard, T., Massot, M., Reveillon, J., and Demoulin, F.X., Where does the droplet size distribution come from?, International Journal of Multiphase Flow, vol. 107, pp. 230-245, 2018.

Deshpande, S.S., Anumolu, L., and Trujillo, M.F., Evaluating the performance of the two-phase flow solver interFoam, Computational Science \& Discovery, vol. 5, no. 1, p. 014016, 2012.

Desjardins, O., Moureau, V., and Pitsch, H., An accurate conservative level set/ghost fluid method for simulating turbulent atomization, Journal of Computational Physics, vol. 227, no. 18, pp. 8395-8416, 2008.

Duret, B., Canu, R., Reveillon, J., and Demoulin, F.X., A pressure based method for vaporizing compressible two-phase flows with interface capturing approach, International Journal of Multiphase Flow, vol. 108, pp. 42-50, 2018.

Duret, B., Luret, G., Reveillon, J., Menard, T., Berlemont, A., and Demoulin, F.X., DNS analysis of turbulent mixing in two-phase flows, International Journal of Multiphase Flow, vol. 40, pp. 93-105, 2012.

Duret, B., Reveillon, J., Menard, T., and Demoulin, F.X., Improving primary atomization modeling 
through DNS of two-phase flows, International Journal of Multiphase Flow, vol. 55, pp. 130-137, 2013.

ECN, https://ecn.sandia.gov/, , Engine Combustion Network.

Gorokhovski, M. and Herrmann, M., Modeling Primary Atomization, Annual Review of Fluid Mechanics, vol. 40, no. 1, pp. 343-366, 2008.

Gueyffier, D., Li, J., Nadim, A., Scardovelli, R., and Zaleski, S., Volume-of-fluid interface tracking with smoothed surface stress methods for three-dimensional flows, Journal of Computational Physics, vol. 152, no. 2, pp. 423 - 456, 1999.

Herrmann, M., A balanced force refined level set grid method for two-phase flows on unstructured flow solver grids, Journal of Computational Physics, vol. 227, no. 4, pp. 2674-2706, 2008.

Hinze, J.O., Fundamentals of the hydrodynamic mechanism of splitting in dispersion processes, AIChE Journal, vol. 1, no. 3, pp. 289-295, 1955.

Kang, M., Fedkiw, R.P., and Liu, X., Boundary conditions capturing method for multiphase incompressible flow, Journal of Scientific Computing, vol. 3, pp. 323-360, 2000.

15 Kindlmann, G., Whitaker, R., Tasdizen, T., and Moller, T., Curvature-based transfer functions for direct volume rendering: Methods and applications, Visualization, 2003. VIS 2003. IEEE, IEEE, pp. 513-520, 2003.

Lalanne, B., Villegas, L.R., Tanguy, S., and Risso, F., On the computation of viscous terms for incompressible two-phase flows with Level Set/Ghost Fluid Method, Journal of Computational Physics, vol. 301, pp. 289-307, 2015.

Lebas, R., Menard, T., Beau, P.A., Berlemont, A., and Demoulin, F.X., Numerical simulation of primary break-up and atomization: DNS and modelling study, International Journal of Multiphase Flow, vol. 35, no. 3, pp. 247-260, 2009.

Liu, X.D., Fedkiw, R.P., and Kang, M., A Boundary Condition Capturing Method for Poisson's Equation on Irregular Domains, Journal of Computational Physics, vol. 160, no. 1, pp. 151 $178,2000$.

Luret, G., Ménard, T., Berlemont, A., Réveillon, J., and Demoulin, F.X., A DNS study ranging from dense to dilute turbulent two-phase flows, 2010.

Ménard, T., Tanguy, S., and Berlemont, A., Coupling level set/VOF/ghost fluid methods: Validation

\section{Preprint version}


and application to $3 \mathrm{~d}$ simulation of the primary break-up of a liquid jet, International Journal of Multiphase Flow, vol. 33, no. 5, pp. 510-524, 2007.

Navarro-Martinez, S., Large eddy simulation of spray atomization with a probability density function method, International Journal of Multiphase Flow, vol. 63, pp. 11 - 22, 2014.

5 Saurel, R. and Pantano, C., Diffuse-Interface Capturing Methods for Compressible Two-Phase Flows, Annual Review of Fluid Mechanics, vol. 50, no. 1, pp. 105-130, 2018.

Shinjo, J. and Umemura, A., Simulation of liquid jet primary breakup: Dynamics of ligament and droplet formation, International Journal of Multiphase Flow, vol. 36, no. 7, pp. 513-532, 2010.

Sussman, M., Smereka, P., and Osher, S., A level set approach for computing solutions to incompressible two-phase flow, Journal of Computational Physics, vol. 114, no. 1, pp. 146 - 159, 1994.

Sussman, M., Smith, K.M., Hussaini, M.Y., Ohta, M., and Zhi-Wei, R., A sharp interface method for incompressible two-phase flows, Journal of Computational Physics, vol. 221, no. 2, pp. 469505, 2007.

15 Thiesset, F., Duret, B., Menard, T., Dumouchel, C., Reveillon, J., and Demoulin, F.X., Liquid transport in scale space, Journal of Fluid Mechanics, vol. 886, p. A4, 2020.

Vallet, A. and Borghi, R., Modélisation eulerienne de l'atomisation d'un jet liquide, Comptes Rendus de l'Académie des Sciences-Series IIB-Mechanics-Physics-Astronomy, vol. 327, no. 10, pp. 1015-1020, 1999.

Vallet, A., Burluka, A.A., and Borghi, R., Development of a Eulerian model for the "Atomization" of a liquid jet, Atomization and Sprays, vol. 11, no. 6, pp. 619-642, 2001.

Vaudor, G., Ménard, T., Aniszewski, W., Doring, M., and Berlemont, A., A consistent mass and momentum flux computation method for two phase flows. Application to atomization process, Computers \& Fluids, vol. 152, pp. 204-216, 2017.

Wardle, K.E. and Weller, H.G., Hybrid Multiphase CFD Solver for Coupled Dispersed/Segregated Flows in Liquid-Liquid Extraction, International Journal of Chemical Engineering, vol. 2013, pp. $1-13,2013$.

Weller, H.G., Tabor, G., Jasak, H., and Fureby, C., A tensorial approach to computational continuum mechanics using object-oriented techniques, Computers in physics, vol. 12, no. 6, pp. 
620-631, 1998.

Preprint version 